\title{
Importancia de los disruptores endocrinos en la alimentación bovina
}

\author{
Importance of endocrine disruptors in food bovine
}

\author{
Jaramillo Hernández Dumar Alexander ${ }^{1}$ \\ ${ }^{1}$ Médico Veterinario Zootecnista, Dipl. Esp., (c)MSc. Docente Universidad de los \\ Llanos. Grupo de Investigación en Farmacología Experimental y Medicina Interna \\ - ÉLITE \\ dumar.jaramillo@unillanos.edu.co
}

Recibido el 30 de Noviembre 2012, Aceptado el 15 de Abril 2013

\begin{abstract}
RESUMEN
En este artículo de revisión se describen las características químicas de los disruptores endocrinos que pueden contaminar los productos utilizados en la alimentación bovina, así mismo su disponibilidad ambiental y los efectos que generan en la homeostasis de esta especie de interés zootécnico, con especial énfasis en las alteraciones que comprometen el sistema reproductor de machos y hembras de la especie bovina; además de las consecuencias en salud pública generadas por la oferta de productos y subproductos de origen bovino con residuos o trazas de disruptores endocrinos. La ecotoxicología juega un papel importante en la explicación de los efectos postinteracción entre los compuestos xenobióticos y los organismos de un determinado ecosistema; las fuentes de disruptores endocrinos se pueden encontrar en los contaminantes orgánicos persistentes como en los fitoestrógenos y éstos pueden estar presentes en los insumos utilizados para balancear las dietas de los animales en producción generando así las alteraciones neuroendocrinas con consecuencias económicas al reducir los parámetros de reproducción del hato.
\end{abstract}

Palabras clave: Disruptor endocrino, seguridad alimentaria, ecotoxicología, contaminante persistente, toxindrome. 


\begin{abstract}
In this review article describes the chemical characteristics of endocrine disruptors that can contaminate food products used in cattle, likewise its environmental availability and the effects they generate in the homeostasis of this species of zoothecnical interest, with special emphasis on alterations that compromise the reproductive system of male and female bovine, besides the public health consequences generated by the supply of products and byproducts of bovine origin with residues or traces of endocrine disruptors. The ecotoxicology plays an important role in explaining the effects post interaction between xenobiotics and the organisms in an ecosystem; the sources of endocrine disruptors can be found on persistent organic pollutants such as phytoestrogens and these can be present on the inputs used to balance the diets of animals in generating production neuroendocrine changes with economic impact by reducing the reproduction parameters herd.
\end{abstract}

Keywords: Endocrine disrupter, food safety, ecotoxicology, persistent contaminant, toxindrome.

\title{
INTRODUCCIÓN
}

El rápido desarrollo de nuevas estructuras materiales y su consumo genera un correspondiente incremento en nuevos productos químicos que interactúan en el ambiente (Weschler, 2009), el principio de la ecotoxicología se basa en las propiedades de acumulación y transferencia de xenobióticos entre el medio ambiente y los organismo que lo habitan, generando procesos de biomagnificación en las cadenas tróficas, teniendo como precedente las consideraciones estructurales de los xenobióticos y su capacidad de perdurar ampliamente sin ser degradados por los procesos implícitos en estos. Los disruptores endocrinos (EDs, por su sigla en inglés) interfieren con la acción de hormonas endógenas, la exposición a estos puede generar efectos adversos en la reproducción y desarrollo productivo, al mostrar disrupción de las señales endocrinas normales tanto en estudios en animales in vitro como in vivo (Colborn et al., 1993). 
La contaminación de los insumos destinados a la formulación de dietas en las producciones bovinas por parte de compuestos ambientales xenobióticos, es una de las mayores preocupaciones de la salud pública veterinaria, además de la bioacumulación de EDs resistentes a temperaturas ambientales que podrían ser fuentes de alteraciones reproductivas tanto en los animales como en los humanos. La Autoridad Europea para la Seguridad Alimentaria (por su sigla en inglés EFSA) delimita y presenta los principales contaminantes de los alimentos en las cadenas productivas del sector primario y extractivo; este ente de control también muestra las sustancias de origen natural, como es el caso de las micotoxinas, como posibles agentes contaminantes de alimentos. Los EDs se dividen en distintos grupos dependiendo de sus orígenes y disponibilidad dentro del sistema de producción animal, por ejemplo, los hallazgos realizados por Kierkegaard et al., (2007) en forrajes utilizados para el pastoreo bovino donde reportan la existencia de retardantes bromados de llama (BFRs), un grupo de compuestos halógenos presentes en plásticos, aislantes eléctricos entre otros productos de consumo; estos BFRs son contaminantes persistentes de alta relevancia en la cadena de alimentos en los cuales también se incluyen los metales pesados (Wilkinson et al., 2003) y especialmente los compuestos clorados, descritos por la EFSA como los insecticidas organoclorados utilizados ampliamente en países industrializados, incluyendo sustancias como el DDT (diclorodifeniltricloroetano) (EFSA, 2006a) y una mezcla conocida como Clordano (EFSA, 2007).

Diferentes publicaciones sugieren que otras fuentes de EDs que pueden contaminar las pasturas son los alquilfenoles, componentes estrógenicos generados por la degradación ambiental de detergentes polietoxilados (Ying et al., 2002); los ptalatos son un grupo importante de plastificadores de resinas de PVC polivinil cloruro (European Union, 2004, 2007; Rudel, 2000), algunos de estos compuestos interfieren con las vías de los diferentes receptores nucleares responsables de los procesos de transcripción dependiente de ligandos hormonales de naturaleza esteroidal, induciendo disturbios endocrinos en animales de laboratorio y posiblemente en humanos (Heudorf et al., 2007). Rhind 
et al., (2005; 2007) reportaron la presencia de nonilfenol (alquilfenol) en leche, hígado, riñón y músculo de ovejas que pastoreaban campos fertilizados con lodos; deduciendo que estos lodos tenían implícitos los productos de lixiviación y escorrentía de suelos donde anteriormente realizaban procesos de lavado con detergentes polietoxilados de instalaciones de otras producciones animales aledañas.

\section{FUENTE, INTERACCIONES Y EFECTOS EN LA SALUD ANIMAL Y HUMANA DE LOS EDs}

\section{Elementos traza y vitaminas, moduladores endocrinos desde la alimentación}

En las listas publicadas para nombrar los EDs no aparecen una serie de elementos traza y vitaminas usadas como aditivos nutricionales en la alimentación animal, estos son importantes moduladores del sistema endocrino, en dado caso que existieran deficiencias o excesos en estos compuestos a nivel de las dietas se presentarían alteraciones endocrinas, ejemplos de estos compuestos son el calciferol, un metabolito primario de la vitamina $D_{3}$ que interactúa con la paratohormona (EFSA, 2005a), el elemento traza selenio, esencial para la función tiroidea (EFSA, 2006a) y las llamadas sales de iodo (EFSA, 2005b). Desde el año 2003 se creó la agencia que estudia y recomienda los aditivos y productos 0 sustancias usadas en la alimentación animal (FEEDAP), este ente recomienda un máximo de $4 \mathrm{mg} / \mathrm{kg}$ iodo en las dietas formuladas para los animales, con excepción de la producción acuícola (20 mg/kg de iodo en el alimento) por la baja presencia de este elemento en los peces marinos, es así que este compuesto tendrá un efecto mitigador de la acción de los compuestos goitrogénicos, como la goitrina presente en una amplia variedad de especies forrajeras, la cual inhibe de manera irreversible la peroxidasa tiroidea originando una disminución sustancial en la producción de tiroxina; además de incrementar el riesgo del bocio en los consumidores de productos o subproductos bovinos provenientes de animales expuestos a estos EDs (EFSA, 2008). 
El cromo muestra una actividad similar, a los electos traza de la dieta, en la función endocrina, está inmiscuido en la homeostasis de la glucosa, en EU el cromo no está autorizado para ser usado como aditivo en los planes de nutrición, aún así diversos autores estudian su rol en la nutrición de los animales de interés zootécnico (Besong et al., 2001; Van de Ligt et al., 2002, Matthews et al., 2003;). El cromo se encuentra naturalmente como un elemento ubicuo, está presente en los organismos y en el medio ambiente, el cromo hexavalente (VI) o cromo industrial genera toxicidad aguda o crónica, a través de la vía dérmica o respiratoria, con efectos carcinogénicos (Sedman et al., 2006), al interior celular el cromo $\mathrm{VI}$ es reducido a cromo trivalente (III), el cual reacciona con proteínas y ácidos nucleícos (Costa y Klein, 2006). El cromo III se encuentra en leche y carne de origen bovino en aproximadamente 0.01 y $0.1 \mathrm{mg} / \mathrm{kg}$, respectivamente (Ysart et al., 2000), en las dietas se encuentra mayor a $1 \mathrm{mg} / \mathrm{kg}$ llegando en algunas instancias a $5 \mathrm{mg} / \mathrm{kg}$ de alimento (Li et al., 2005a). El cromo III es un cofactor para la insulina (Borel y Anderson, 1984), es transportado por la transferrina en el plasma y juega un papel importante con la toma de la glucosa en los tejidos (Morris et al., 1993), al parecer el cromo III organiza una oligoproteína citoplasmática, la cromomodulina, este compuesto incrementa la captación de glucosa por parte de la célula al elevar la sensibilidad del transportador de glucosa, Glut 4 (Chen et al., 2005). En rumiantes el cromo III exhibe un incremento en plasma de colesterol y triglicéridos, reduciendo las lipoproteínas de alta densidad y la no esterificación de ácidos grasos (Bunting et al., 2000; Kegley et al., 2000; McNamara y Valdez, 2005); también el cromo III está implicado en la modulación de las respuestas del sistema inmune, pero sin una función específica (Kegley et al., 1996; Van Heugten y Spears, 1997; Shrivastava et al., 2002).

Se debe tener presente los requerimientos nutricionales de los animales para no generar alteraciones en los niveles de los elementos traza y las vitaminas, estos compuestos suelen estar presentes en los planes de alimentación de los bovinos. En los párrafos anteriores se presentaron las actividades biológicas de este tipo de compuestos donde tienen una alta relevancia en diferentes reacciones 
bioquímicas y fisiológicas, para mantener la homeostasis, en ese orden de ideas la función reproductiva está basada en la correcta disposición de sustratos para la obtención de los productos necesarios para el óptimo funcionamiento del eje hipotálamo-hipófisis-gónadas, donde el cromo III, las sales iodadas y las vitaminas entran a modular las actividades de este eje neuroendocrino.

\section{Bifenilos policlorados (PCBs)}

En el año de 1980 se produjo, en los países industrializados, más de 1 millón de toneladas de productos que contenían PCBs (Figura 1. ejemplo de bifenilos policlorados) por parte de la industria y el sector comercial, estos compuestos se encontraban principalmente en equipos eléctricos y sistemas hidráulicos; el ingreso al medio ambiente se generaba en la disposición incorrecta a la cual eran sometidos, ofertando productos persistentes en el ambiente originando problemas globales de contaminación (ESFA, 2005). Los PCBs comprenden 209 congéneres diferentes divididos en dos grupos acorde con sus propiedades toxicológicas. La PBCs-12'semejante a dioxina, que interactúa con el receptor aril-hidrocarbono (AhR; "receptor dioxinico"), proteína intracitoplasmática que funciona como receptor de hormonas esteroidales, y los PCBs no semejantes a dioxinas, los cuales eventualmente difieren en toxicidad y persistencia (EFSA, 2005b; La Rocca et al., 2006). Varios estudios de casos han reportado la presentación de toxindromes por PCBs a través de la alimentación en animales de interés zootécnico, cuando accidentalmente los PCBs superan los $30 \mathrm{mg} / \mathrm{kg}$ en la dieta suministrada los efectos generados principalmente están enmarcados en alteraciones del tracto gastrointestinal y alteraciones evidentes en la reproducción del hato bovino (EFSA, 2005), la contaminación de forrajes por material particulado generado en procesos de incineración de desechos (Martí-Cid et al., 2008) y las materias primas utilizadas en la formulación de las dietas son las fuentes primarias de los PCBs en las producciones bovinas (EFSA, 2005c; La Rocca et al., 2006). 


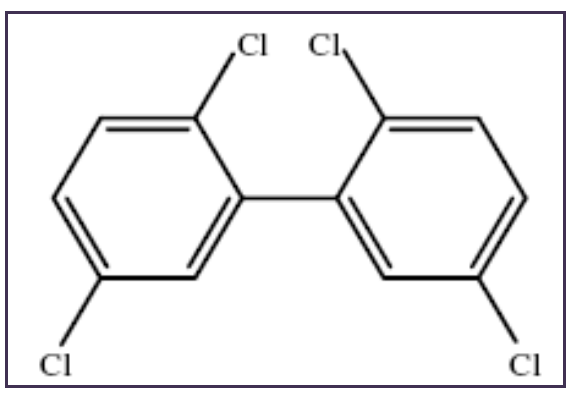

Figura 1. Estructura molécular del 2,2`,5,5`-Tetraclorobifenil (PCB 52) Modificado de: Rudel y Perovich, (2009)

La bioacumulación ocurre en tejido adiposo, hígado y músculo (grasa existente entre fibras musculares), así mismo los PCBs son transferidos a los productos y subproductos generados de los animales expuestos a estos EDs, por ejemplo, la leche, en donde este tipo de xenobiótico se encuentran asociados a los cuerpos grasos (Thomas et al., 1999). Aunque documentos generados por EFSA, (2005) comentan sobre la alta tolerancia de los bovinos a la exposición de PCBs en los cuales no se desarrollan toxindromes letales, siendo esto una dificultad para identificar la población en riesgo; en Brescia (Norte de Italia) se originó la contaminación de pasturas con PCBs provenientes de los residuos líquidos de fábricas que vertían sus efluentes en campos, generando los procesos de biomagnificación y exposición a los bovinos a través de los ácidos grasos presentes en los forrajes, encontrando en la población humana que consumía los productos y subproductos provenientes de los bovinos expuestos niveles de PCBs de $140 \mathrm{ng} / \mathrm{kg}$ de peso corporal (Donato et al., 2006; Turrio Baldassarri et al., 2007), evidenciando la capacidad de este tipo de EDs de ser transmitido a través de los sistemas productivos hasta la canasta familiar.

Los PCBs son promotores de neoplasias, sobre todo a nivel hepático, específicamente los congéneres PBCs-12'semejante a dioxina, inducen estrés oxidativo y/o inhibición de la apoptosis (Tharappel et al., 2002); así mismo los PCBs no semejantes a dioxinas generan una fuerte inducción de la actividad del citocromo-P450 (Strathmann et al., 2006). Las personas que consumen productos de origen animal con residuos PCBs, pueden desarrollar polimorfismo de genes 
codificantes de enzimas biotransformantes, especialmente las mujeres posmenopáusicas predispuestas a sufrir cáncer de mama por el polimorfismo de CYP1A1, esta enzima promueve la formación PCB hidroximetabolitos los cuales tienen una acción estrogénica indirecta por la inhibición de la enzima sulfatransferasa responsable por la detoxificación del estradiol, desarrollándose un aumento de este estrógeno en sangre con la subsiguiente aparición de neoplasias dependientes de estos compuestos (Kester et al., 2000; Li et al., 2005b). La glándula tiroides también puede ser blanco de los PCBs, en estudios realizados en ratas, el congénere PCB 126 generó interrupción del metabolismo hepático de la tiroxina, así mismo de la regulación existente en el eje hipotálamo-pituitariatiroides (Fisher et al., 2006), al parecer el mecanismo de acción ligado a estas alteraciones se basa en el desplazamiento de la proteína transtiretina, transportadora de la hormona tiroxina, por parte de los PCB hidroximetabolitos (Lans et al., 1994), además del cambio en el catabolismo de la tiroxina en el hígado por incremento de la actividad de enzimas glocoroniltransferasas (Vansell y Klaassen, 2002).

\section{Retardantes bromados de llama (BFRs)}

Los BFRs pueden activar las vías del receptor nuclear generando aumento en la expresión de los genes de las monooxidasas microsomales hepáticas CYP1A1, CYP2B y CYP3A, regulando esta actividad a través del AhR, además de los receptores para el androstano y pregnano (Sanders et al., 2005; Peters et al., 2006; Pacyniak et al., 2007).

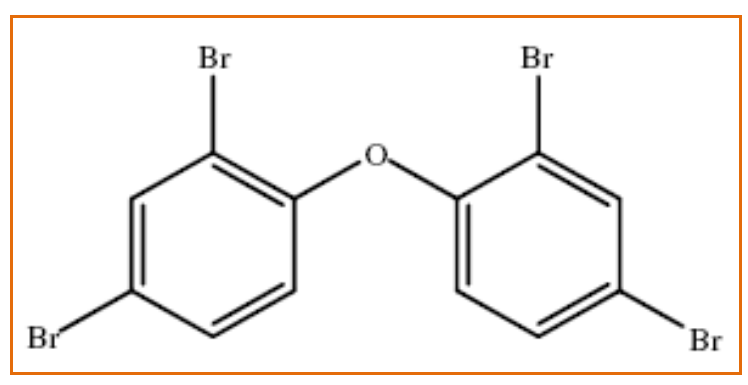

Figura 2. Estructura molécular del 2,2,4,4-tetrabromodifenileter (BDE 47) Modificado de: Rudel y Perovich, (2009) 
Por estas razones la EFSA incluye en sus programas de monitorización de seguridad alimentaria estos productos (EFSA, 2006b), aunque se cuenta con muy poca información sobre sus efectos, existen reportes de una contaminación accidental de alimentos con compuestos bromados en un hato bovino de Michigan en el año de 1973, el compuesto de mayor interés en esta intoxicación masiva fue el bifenilo policromado (Figura 2, ejemplo de retardante bromado de llama), los signos clínicos observados en los animales fueron: emaciación progresiva, hiperqueratosis y daño renal (Fries, 1985); mientras que en estudios realizados en consumidores de productos de origen bovino con residuos de BFRs se identifico la presentación de disfunciones inmunes (Bekesi et al., 1987) y un incremento en los pesos al nacimiento de infantes expuestos in-útero a estos EDs. (Sweeney et al., 2007).

\section{EDs derivados de plantas}

Los EDs derivados de plantas son otro grupo de contaminantes de alimentos, unos de los ejemplos más representativos es la micotoxina de características estrógenicas conocida como zearalenona (EFSA, 2004) y los glucosinolatos derivados de los isoticionatos (EFSA, 2008). Estos EDs son reconocidos como potenciales compuestos que afectan la producción animal, los isoticionatos son conocidos por sus capacidades goitrogénicas, al inhibir la captación de iodo por parte de la glándula toriodes, desarrollando una baja iodinización de los residuos de tirosina de la tiroglobulina (EFSA, 2008). La zearalenona presenta alteraciones endocrinas dependientes de las consideraciones etarias, siendo más susceptibles los bovinos sexualmente inmaduros, el riesgo principal para la exposición se debe al incorrecto almacenamiento de materias primas de la dieta, particularmente el maíz, aumentando la humedad y temperatura del alimento creciendo en este el hongo Fusarium spp, productor de la zearalenona (Zinedine et al., 2007).

\section{Pesticidas}

La mayor clase de pesticidas incluyen organoclorados como el DDT y clordano (Figura 3, ejemplo de pesticida organoclorado); organofosforados como el 
clorpirifos; carbamatos como el carbaril; y piretroides como la permetrina; estos productos también vienen conjugados con antimicrobianos, por ejemplo, el triclosan y triclocarban y O-fenil fenol. La vía de ingreso a los bovinos es a través de la piel intacta por las prácticas de aspersiones insecticidas y por vía oral en los forrajes, estas plantas destinadas al consumo se exponen por lixiviación y/o escorrentía a interacciones con estos EDs (McKinlay et al., 2008).

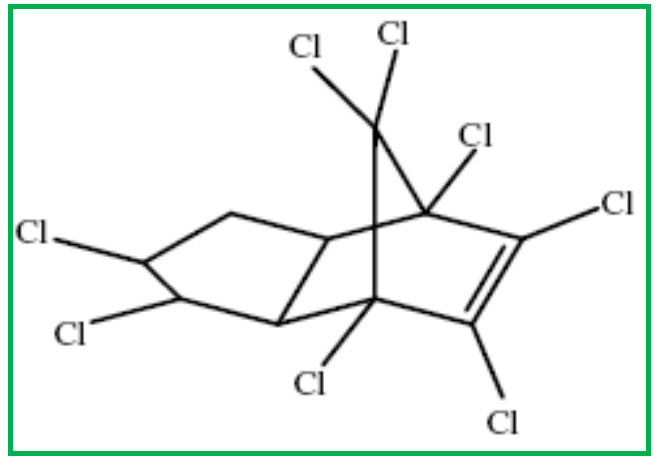

Figura 3. Estructura molécular del clordano Modificado de: Rudel y Perovich, (2009)

Los pesticidas son identificados como EDs (Kelce et al., 1995; Soto et al., 1994; Soto et al., 1995), generan una variedad de alteraciones orgánicas difiriendo de acuerdo a sus propiedades toxicodinámicas, sus efectos se observan en desórdenes de la lactación y predisposición a cáncer (Kilburn y Thornton, 1995; Brody et al., 2007; Cohn et al., 2007; Rogan y Ragan, 2007), también se adicionan a esta lista las alteraciones neuroendocrinas como es el caso de los piretroides, que poseen un efecto antiandrogénico, antiestrógenico o actividad estrogénica, y el clorpirifos que muestra un efecto sobre la actividad hormonal tiroidea de humanos y animales (Garey y Wolff, 1998; Go et al., 1999; Adibi et al., 2003; Kim et al., 2005a,b; Jeong et al., 2006; Meeker et al., 2006).

Queda claro que la contaminación de los alimentos utilizados en los planes de manejo de las producciones bovinas, con disruptores endocrinos, no es una nueva faceta de la ecotoxicología veterinaria, ya que existen reportes de efectos estrógenicos del trébol rojo en ovejas desde el año 1950 (Garner, 1961; Adams, 1998), sin embargo, la situación global muestra el avance en la maximización de 
las producciones animales, introduciendo diferentes insumos y aditivos en los planes de alimentación, implementando un manejo intensivo del suelo con base en productos artificiales que permitan una mayor producción de forrajes y otros cultivos que funcionan como insumos en la alimentación animal; lo anterior implica una alta predisposición a originar la exposición de los animales de interés zootécnico a los EDs, así mismo genera una amplia susceptibilidad de disminuir la calidad y seguridad alimentaria con productos de origen animal para el consumo humano, agudizando la problemática de salud pública.

Así mismo, la minimización del impacto sobre el ambiente a partir de estrategias congruentes con la sostenibilidad y seguridad alimentaría serán el sustento de la magnificación de los parámetros productivos de las empresas ganaderas. Un ejemplo de estos procesos puede ser la introducción de fuentes nutricionales confiables, libres de contaminación (EFSA, 2008), además de los estudios contextualizados sobre los requerimientos nutricionales de los animales bovinos utilizados en la producción, teniendo en cuenta tasas metabólicas, selección genética desde el punto de vista vulnerabilidad del sistema endocrino, especialmente en animales gestantes, lactantes y en bovinos de reemplazo (Kegley et al., 2000; Shrivastava et al., 2002).

\section{CONCLUSIONES}

Es necesario evaluar de manera cualitativa y cuantitativa la incidencia de los EDs en la producción bovina del país, tomando como base las posibles fuentes de estos compuestos bioactivos, generando teorías de interrelación ambientalorganismos (principios de ecotoxicología), implementando sistemas de evaluación de contaminación ambiental en los diferentes procesos industriales y comerciales del las regiones en general, permitiendo poseer un conocimiento avanzado de los EDs y su relación ambiental, contextualizado en las medidas de control y minimización del impacto de estos sobre los sistemas productivos animales.

También es necesario estimar la posible exposición de los consumidores humanos a los EDs, desde el estudio de los puntos de riesgo de contaminación en la 
cadena productiva, para esto, se deben organizar, entre los estamentos gubernamentales (ICA, INVIMA, UMATAS, entre otros) y privados (universidades, laboratorios farmacéuticos, plantas de concentrados, entre otras), planes de contingencia y mejoramiento continuo referente a la optimización de la seguridad y calidad alimentaria a lo largo del ciclo productivo y comercialización de productos y subproductos animales; es decir realizar proyectos marco de valoración de la exposición ambiental a EDs, mapas de población en riesgo (Ohh y Lee, 2005), análisis químico pertinente a la evaluación continuo de insumos y productos utilizados en la producción animal, con la finalidad de reducir la exposición fuentes de EDs.

La actividad de los disruptores endocrinos en la homeostasis es amplia, incluyendo la interferencia con los procesos de regulación y/o desarrollo de vías enzimáticas-bioquímicas (Muto et al., 2002; Sanders et al., 2005; Bennett et al., 2006; Pacyniak et al., 2007), el entendimiento apropiado de los procesos implícitos en los toxindromes generados por EDs, permitirá fundamentar el conocimiento en la asociación entre los efectos en la salud y las exposiciones en la dieta tanto de animales como humanos. En este orden de ideas implementar estudios con biomarcadores para conocer los caminos y procesos de detoxificación (toxicocinética) a los que son sometidos los EDs (Fernández et al., 2007), clarificarían los posibles tratamientos generales a instaurar en las producciones animales y poblaciones humanas para mitigar los efectos a futuro de los EDs; así mismo la proteómica es una de las estrategias que muestra avances significativos en la caracterización de biomarcadores que serían susceptibles de uso en los animales de interés zootécnico (Gardini et al., 2006).

Son necesarios más estudios sobre los contaminantes orgánicos persistentes en la geografía colombiana, existen estudios en otros países que tienden a valorar con precisión las implicaciones de los EDs en la producción animal, ejemplos de estos son el cromo (III) como componente basal en las dietas para animales (Li et al., 2005a), la bioacumulación de los BFRs (Hites et al., 2004; Kierkegaard et al., 2007) y la presencia de ptalatos como contaminantes presentes en plásticos 
(Rhind et al., 2005, 2007). En Colombia no existen reportes sobre la evaluación de los efectos de los EDs en las producciones animales, sus posibles fuentes, interacciones, procesos de biomagnificación y estudios ecotoxicológicos que permitan desde esta base generar estrategias consolidadas para mitigar las consecuencias en salud pública y ser competitivos comercialmente. Existen estudios globales sobre contaminantes emergentes que tienen que ser valorados en las producciones bovinas, ejemplo de ello son los componentes perfluorinados (Ericson et al., 2008) o metales del grupo del platino (Frazzoli et al., 2007), en este orden de ideas la generación de base de datos que organicen la información existente de manera específica para tener una divulgación nacional e internacional que permita generar discusiones entorno a los EDs; en el mundo las organizaciones científicas tratan de esclarecer y recoger toda la información generada respecto a los compuestos xenobióticos bioactivos, su tarea es insuficiente, se cuenta con el Comité Científico sobre los Alimentos (2000), los reportes realizados por a EFSA (2005) sobre los PCB no semejantes a dioxinas; sin embargo estas organizaciones no cuentan con una información completa por la falta de estudios científicos aptos y claros sobre las repercusiones de los EDs en las producciones animales y poblaciones humanas.

\section{REFERENCIAS BIBLIOGRÁFICAS}

1. Adams N. R. Natural and anthropogenic environmental oestrogens: the scientific basis for risk assessment, Clover phyto-oestrogens in sheep in Western Australia. Pure and Applied Chemistry, 70: 1855-1862. 1998.

2. Adibi J. J., Perera F. P. et al. Prenatal exposures to phthalates among women in New York City and Krakow, Poland. Environmental Health Perspectives, 111 (14): 1719-1722. 2003.

3. Bekesi J. G., Roboz J. P., Fischbein A., Mason, P. Immunotoxicology: environmental contamination by polybrominated biphenyls and immune dysfunction among residents of the State of Michigan. Cancer Detection and Prevention, (Suppl. 1): 29-37. 1987.

4. Bennett R., Adams B., French A., Neggers Y., Vincent J. B. High-dose chromium (III) supplementation has no effects on body mass and composition while altering plasma hormone and triglycerides concentrations. Biological Trace Element Research, 113: 53-66. 2006.

5. Besong S., Jackson J. A., Trammell D. S., Akay V. Influence of supplemental chromium on concentrations of liver triglyceride, blood metabolites and rumen VFA 
profile in steers fed a moderately high fat diet. Journal of Dairy Science, 84: 1679_ 1685. 2001.

6. Borel J. S., Anderson R. A. Chromium. In: Biochemistry of the Essential Ultratrace Elements. Plenum Press, New York, pp. 175-199. 1984.

7. Brody J. G., Moysich K. B. et al. Environmental pollutants and breast cancer: epidemiologic studies. Cancer, 109 (12 Suppl): 2667-2711. 2007.

8. Bunting L. D., Tarifa T. A., Crochet B. T., Fernandez J. M., Depew C. L., Lovejoy J. C. Effects of dietary inclusion of chromium propionate and calcium propionate on glucose disposal and gastrointestinal development in dairy calves. Journal of Dairy Science, 83: 2491-2498. 2000.

9. Colborn, T., VomSaal, F., et al. Developmental effects of endocrine-disrupting chemicals in wildlife and humans. Environmental Health Perspectives, 101 (5): 378-385. 1993.

10. Cohn B. A., Wolff M. S., et al. DDT and breast cancer in young women: new data on the significance of age at exposure. Environmental Health Perspectives, 115 (10): 1406-1414. 2007.

11. Chen G., Liu P., Pattar G. R., Tackett L., Bhonagiri P., Strawbridge A. B., Elmendorf J. S. Chromium activates glucose transporter 4 trafficking and enhances insulin-stimulated glucose transport in 3T3-L1 adipocytes via cholesteroldependent mechanism. Molecular Endocrinology, 20: 857-870. 2005.

12. Costa M., Klein B. Toxicity and carcinogenicity of chromium compounds in humans. Critical Reviews in Toxicology, 36: 155-163. 2006.

13. Donato F., Magoni M., Bergonzi R., Scarcella C., Indelicato A., Carasi S., Apostoli $P$. Exposure to polychlorinated biphenyls in residents near a chemical factory in Italy: the food chain as main source of contamination. Chemosphere, 64: 15621572. 2006.

14. Ericson I., Martí-Cid R., Nadal M., Van Bavel B., Lindström G., Domingo J. L., Human exposure to perfluorinated chemicals through the diet: intake of perfluorinated compounds in foods from the Catalan (Spain) market. Journal of Agricultural and Food Chemistry, 56: 1787-1794. 2008.

15. European Food Safety Authority. Opinion of the scientific panel on contaminants in the food chain on a request from the commission related to zearalenone as undesirable substance in animal feed, July 2004. Recuperado 21 Abril 2008. Disponible

En: http://www.efsa.europa.eu/en/science/contam/contam opinions/527.html

16. European Food Safety Authority. Opinion of the FEEDAP Panel on the evaluation of safety and efficacy of 'Hy_D' (calcifediol), based on 25hydroxylcholecalciferol/25-hydroxy-pre-cholecalciferol, as feed additive in accordance with Council Directive 70/524/EEC, Mayo 2005a. Recuperado 21 Abril 2008. Disponible http://www.efsa.europa.eu/en/science/feedap/feedap opinions/994.html

17. European Food Safety Authority. Opinion of the scientific panel on contaminants in the food chain on a request from the commission related to the presence of NonDioxin-Like Polychlorinated Biphenyls (PCB) in feed and food, Noviembre 2005b. Recuperado 212008.2 Abril 21 En: http://www.efsa.europa.eu/en/science/contam/contam opinions/1229.html

18. European Food Safety Authority. Opinion of the scientific panel on contaminants in the food chain on a request from the commission related to the presence of Non- 
Dioxin-Like Polychlorinated Biphenyls (PCB) in feed and food, Noviembre 2005c. Recuperado $21 \quad$ Abril $2008 . \quad$ Disponible En: http://www.efsa.europa.eu/en/science/contam/contam opinions/1229.html

19. European Food Safety Authority. Opinion of the Scientific Panel CONTAM related to DDT as an undesirable substance in animal feed, November 2006a. Recuperado $21 \quad$ Abril 2008.2 Disponible En: http://www.efsa.europa.eu/en/science/contam/contam opinions/eh433 ddt.html

20. European Food Safety Authority. Advice of the scientific panel on contaminants in the food chain on a request from the commission related to relevant chemical compounds in the group of brominated flame retardants for monitoring in feed and food, February 2006. Recuperado 21 Abril 2008. Disponible En: http://www.efsa.europa.eu/en/science/contam/contam documents/1380.html

21. European Food Safety Authority. Scientific opinion of the panel on contaminants in the food chain on chlordane as undesirable substance in animal feed, November 2007. Recuperado 21 Abril 2008. Disponible En: http://www.efsa.europa.eu/EFSA/efsa locale$11786207538121178661055358 . \mathrm{htm}$

22. European Food Safety Authority. Opinion of The Scientific Panel on Contaminants in the Food Chain on Glucosinolates as undesirable substances in animal feed Scientific Opinion of the Panel on Contaminants in the Food Chain, November 2007. Recuperado 21 Abril 2008. Disponible En: http://www.efsa.europa.eu/EFSA/efsa locale$11786207538121178678427782 . \mathrm{htm}$

23. European Union. Risk Assessment Report: dibutyl phthalate. European Chemicals Bureau, The Netherlands. 2004.

24. European Union. Risk Assessment Report: benzylbutyl phthalate. European Chemicals Bureau, Norway. 2007.

25. Fernández M. F., Santa-Marina L., Ibarluzea J. M., Exposito J., Aurrekoetxea J. J., Torne P., Laguna J., Rueda A. I., Pedraza V., Olea N. Analysis of population characteristics related to the total effective xenoestrogen burden: a biomarker of xenoestrogen exposure in breast cancer. European Journal of Cancer, 43: 12901299. 2007.

26. Frazzoli C., Cammarone R., Caroli S. Investigation of palladium and platinum levels in food by sector field inductively coupled plasma mass spectrometry. Food Additives and Contaminants, 24: 546-552. 2007.

27. Gardini G., Del Boccio P., Colombatto S., Testore G., Corpillo D., Di llio C., Urbani A., Nebbia C. Proteomic investigation in the detection of the illicit treatment of calves with growth-promoting agents. Proteomics, 6: 2813-2822. 2006.

28. Garey J., Wolff M. S. Estrogenic and antiprogestagenic activities of pyrethroid insecticides. Biochemical and Biophysical Research Communications, 251 (3): 855-859. 1998.

29. Garner R. J. Veterinary Toxicology, second ed. Baillière, Tindall and Cox, London. pp. 358. 1961.

30. Go V., Garey J. et al., Estrogenic potential of certain pyrethroid compounds in the MCF-7 human breast carcinoma cell line. Environmental Health Perspectives, 107 (3): 173-177. 1999. 
31. Heudorf U., Mersch-Sundermann V., Angerer J. Phthalates: toxicology and exposure. International Journal of Hygiene and Environmental Health, 210: 623624. 2007.

32. Hites R. A., Foran J. A., Schwager S. J., Knuth B. A., Hamilton M. C., Carpenter D. $O$. Global assessment of polybrominated diphenyl ethers in farmed and wild salmon. Environmental Science Technology, 38: 4945-4949. 2004.

33. Jeong S. H., Kim B. Y., et al. Effect of chlorpyrifos-methyl on steroid and thyroid hormones in rat F0- and F1-generations. Toxicology, 220 (2-3): 189-202. 2006.

34. Fisher J. W., Campbell J., Muralidhara S., Bruckner J. V., Ferguson D., Mumtaz M., Harmon B., Hedge J. M., Crofton K. M., Kim H., Almekinder T. L. Effect of PCB 126 on hepatic metabolism of thyroxine and perturbations in the hypothalamicpituitary-thyroid axis in the rat. Toxicological Sciences, 90: 87-95. 2006.

35. Fries G. F. The PBB episode in Michigan: an overall appraisal. Critical Reviews in Toxicology, 16: 105-156. 1985.

36. Kegley E. B., Spears J. W., Brown T. T. Immune response and disease resistance of calves fed chromium nicotinic acid complex or chromium chloride. Journal of Dairy Science, 79: 1278-1283. 1996.

37. Kegley E. B., Galloway D. L., Fakler T. M. Effect of dietary chromium-Lmethionine on glucose metabolism of beef steers. Journal of Animal Science, 78: 3177-3183. 2000.

38. Kester M. H., Bulduk S., Tibboel D., Meinl W., Glatt H., Falany C. N., Coughtrie M. W., Bergman A., Safe S. H., Kuiper G. G., Schuur A. G., Brouwer A., Visser T. J. Potent inhibition of estrogen sulfotransferase by hydroxylated PCB metabolites: a novel pathway explaining the estrogenic activity of PCB. Endocrinology, 141: 1897-1900. 2000.

39. Kierkegaard A., Asplund L., de Wit C. A., McLachlan M. S., Thomas G. O., Sweetman A. J., Jones K. C. Fate of higher brominated PBDEs in lactating cows. Environmental Science Technology, 41: 417-423. 2007.

40. Kilburn K. H., Thornton J. C. Protracted neurotoxicity from chlordane sprayed to kill termites. Environmental Health Perspectives, 103 (7-8): 690-694. 1995.

41. Kim S. S., Kwack S. J., et al. Assessment of estrogenic and androgenic activities of tetramethrin in vitro and in vivo assays. Journal of Toxicology and Environmental Health, Part A 68 (23-24): 2277-2289. 2005a.

42. Kim S. S., Lee R. D., et al., Potential estrogenic and antiandrogenic effects of permethrin in rats. The Journal of Reproduction and Development, 51 (2): 201210. 2005b.

43. Lans M. C., Spiertz C., Brouwer A., Koeman J. H. Different competition of thyroxine binding to transthyretin and thyroxine-binding globulin by hydroxyPCBs, PCDDs and PCDFs. European Journal of Pharmacology, 270: 129-136. 1994.

44. La Rocca C., Mantovani A. From environment to food: the case of PCB. Annali dell'Istituto Superiore di Sanità, 42: 410-416. 2006.

45. Li Y., McCroy P. D. J., Saam H., Jackson S. D. A survey of selected heavy metal concentration in Wisconsin dairy feeds. Journal of Dairy Science, 88: 2911-2922. 2005a.

46. Li Y., Millikan R. C., Bell D. A., Cui L., Tse C. K., Newman B., Conway K. Polychlorinated biphenyls, cytochrome P450 1A1 (CYP1A1) polymorphisms, and breast cancer risk among African American women and white women in North 
Carolina: a population-based case-control study. Breast Cancer Research, 7: 1218. 2005b.

47. McNamara J. P., Valdez F. Adipose tissue metabolism and production responses to calcium propionate and chromium propionate. Journal of Dairy Science, 88: 2498-2507. 2005.

48. Martí C. R., Bocio A., Domingo J. L. Dietary exposure to PCDD/PCDFs by individuals living near a hazardous waste incinerator in Catalonia, Spain: temporal trend. Chemosphere, 70: 1588-1595. 2008.

49. Matthews J. O., Higbie A. D., Southern L. L., Coombs D. F., Bidner T. D., Odgaard R. L. Effect of chromium propionate and metabolizable energy on growth, carcass traits, and pork quality of growing-finishing pigs. Journal of Animal Science, 81: 191-196. 2003.

50. Meeker J. D., Barr D. B. et al., Thyroid hormones in relation to urinary metabolites of non-persistent insecticides in men of reproductive age. Reproductive Toxicology, 22 (3): 437-442. 2006.

51. Morris B. W., Gray T. A., MacNeil S. Glucose-dependent uptake of chromium in human and rat insulin-sensitive tissues. Clinical Chemistry, 84: 477-482. 1993.

52. Muto T., Wakui S., Imano N., Nakaaki K., Takahashi H., Hano H., Furusato M., Masaoka T. Mammary gland differentiation in female rats after prenatal exposure to 3,30,4,40,5-pentachlorobiphenyl. Toxicology, 177: 197-205. 2002.

53. Ohh S. J., Lee J. Z. Dietary chromium-methionine chelate supplementation and animal performance. Asian-Australasian Journal of Animal Sciences, 18: 898-907. 2005.

54. Pacyniak E. K., Cheng X., Cunningham M. L., Crofton K., Klaassen C. D., Guo G. $\mathrm{L}$. The flame retardants, polybrominated diphenyl ethers, are pregnant $\mathrm{X}$ receptor activators. Toxicological Sciences, 97: 94-102. 2007.

55. Peters A. K., Nijmeijer S., Gradin K., Backlund M., Bergman A., Poellinger L., Denison M. S., Van den Berg M. Interactions of polybrominated diphenyl ethers with the aryl hydrocarbon receptor pathway. Toxicological Sciences, 92: 133-142. 2006.

56. Rhind S. M., Kyle C. E., Telfer G., Duff E. I., Smith A. Alkyl phenols and diethylhexyl phthalate in tissues of sheep grazing pastures fertilized with sewage sludge or inorganic fertilizer. Environmental Health Perspective, 113: 447-453. 2005.

57. Rhind S. M., Kyle C. E., Mackie C., Telfer G. Effects of exposure of ewes to sewage sludge-treated pasture on phthalate and alkyl phenol concentrations in their milk. Science of the Total Environment, 383: 70-80. 2007.

58. Rogan W. J., Ragan N. B. Some evidence of effects of environmental chemicals on the endocrine system in children. International Journal of Hygiene and Environmental Health, 210 (5): 659-667. 2007.

59. Rudel R. Polycyclic aromatic hydrocarbons, phthalates. En: Spengler J. D., Samet J. M., McCarthy J. F. (Eds.), Indoor Air Quality Handbook. McGraw-Hill, New York phenols. 2000.

60. Sanders J. M., Burka L. T., Smith C. S., Black W., James R., Cunningham, M. L. Differential expression of CYP1A, 2B, and 3A genes in the F344 rat following exposure to a polybrominated diphenyl ether mixture or individual components. Toxicological Sciences, 88: 127-133. 2005. 
61. Sedman M., Beaumont J., McDonald T. A., Reynolds S., Krowech G., Howd R. Review of the evidence regarding the carcinogenicity of hexavalent chromium in drinking water. Journal of Environmental Science and Health, Part C 24: 155-182. 2006.

62. Scientific Committee on Food. Opinion of the scientific committee on food on the tolerable upper intake level of manganese. 2000. Disponible En: http://ec.europa.eu/food/fs/sc/scf/out80f en.pdf

63. Shrivastava R., Upreti R., Seth P., Chaturvedi U., Effects of chromium on the immune system. FEMS Immunology and Medical Microbiology, 34: 1-7. 2002.

64. Strathmann J., Schwarz M., Tharappel J. C., Glauert H. P., Spear B. T., Robertson L. W., Appel K. E., Buchmann A. PCB 153, a non-dioxin-like tumor promoter, selects for beta-catenin (Catnb)-mutated mouse liver tumors. Toxicological Sciences, 93: 34-40. 2006.

65. Sweeney A. M., Symanski E. The influence of age at exposure to PBBs on birth outcomes. Environmental Research, 105: 370-379. 2007.

66. Tharappel J. C., Lee E. Y., Robertson L. W., Spear B. T., Glauert H. P. Regulation of cell proliferation, apoptosis, and transcription factor activities during the promotion of liver carcinogenesis by polychlorinated biphenyls. Toxicological Applied Pharmacology, 179: 172-184. 2002.

67. Thomas G. O., Sweetman A. J., Jones K. C. Input-output balance of polychlorinated biphenyls in a long-term study of lactating dairy cows. Environmental Science Technology, 33: 104-112. 1999.

68. Turrio B. L., Abate V., Alivernini S., Battistelli C. L., Carasi S., Casella M., lacovella N., lamiceli A. L., Indelicato A., Scarcella C., La Rocca C. A study on PCB, PCDD/PCDF industrial contamination in a mixed urban-agricultural area significantly affecting the food chain and the human exposure. Part I: soil and feed. Chemosphere, 67: 1822-1830. 2007.

69. Van de Ligt C. P., Lindemann M. D., Cromwell G. L. Assessment of chromium tripicolinate supplementation and dietary protein level on growth, carcass, and blood criteria in growing pigs. Journal of Animal Science, 80: 2412-2419. 2002.

70. Van Heugten E. V., Spears J. W. Immune response and growth of stressed weanling pigs fed diets supplemented with organic or inorganic forms of chromium. Journal of Animal Science, 75: 409-416. 1997.

71. Vansell N. R., Klaassen C. D. Increase in rat liver UDP-glucuronosyltransferase mRNA by microsomal enzyme inducers that enhance thyroid hormone glucuronidation. Drug Metabolism and Disposition, 30: 240-246. 2002.

72. Weschler C. Changes in indoor pollutants since the 1950s. Atmospheric Environment, 43 (1): 153-169. 2009.

73. Wilkinson J. M., Hill J., Phillips C. J. The accumulation of potentially-toxic metals by grazing ruminants. Proceedings of the Nutrition Society, 6: 267-277. 2003.

74. Ying G. G., Williams B., Kookana R. Environmental fate of alkylphenols and alkylphenol ethoxylates - a review. Environment International, 28: 215-226. 2002.

75. Ysart G., Miller P., Croasdlae M., Crews H., Robb P., Baxter M., de L'Argy C., Harrison N. UK total diet study - dietary exposures to aluminium, arsenic, cadmium, chromium, copper, lead, mercury, nickel, selenium, tin and zinc. Food Additives and Contaminants, 17: 775-786. 2000. 